\title{
Effects of voluntary exercise on blood pressure, angiotensin II, aldosterone, and renal function in two-kidney, one-clip hypertensive rats
}

This article was published in the following Dove Press journal: Integrated Blood Pressure Control

\author{
Brian MWaldman ${ }^{1,2}$ \\ Robert A Augustyniak ${ }^{1-3}$ \\ Haiping Chen ${ }^{1,2}$ \\ Noreen F Rossi ${ }^{1,2,4}$ \\ 'Department of Internal Medicine, \\ ${ }^{2}$ Department of Physiology, \\ Wayne State University School of \\ Medicine, Detroit, MI, ${ }^{3}$ Department \\ of Biomedical Sciences, Edward \\ Via College of Osteopathic \\ Medicine-Carolinas, Spartanburg, \\ SC, ${ }^{4}$ Department of Internal \\ Medicine, John D Dingell Veterans \\ Administration Medical Center, \\ Detroit, MI, USA
}

\begin{abstract}
Spontaneous dynamic exercise promotes sympathoinhibition and decreases arterial pressure in two-kidney, one-clip (2K-1C) hypertensive rats. Renal sympathetic nerves stimulate renin secretion and increase renal tubular sodium reabsorption. We hypothesized that daily voluntary wheel running exercise by $2 \mathrm{~K}-1 \mathrm{C}$ rats will decrease mean arterial pressure (MAP), plasma angiotensin II (Ang II), and aldosterone as well as normalize urinary sodium and potassium excretion independent of changes in glomerular filtration rate (GFR). Five-week-old male Sprague Dawley rats underwent sham clipping (Sham) or right renal artery clipping (2K-1C). Rats were randomized to standard caging (SED) or cages with running wheels (EX). After 12 weeks, rats were assigned to either collection of aortic blood for measurement of Ang II and aldosterone or assessment of inulin clearances and excretory function. Running distances were comparable in both EX groups. MAP was lower in $2 \mathrm{~K}-1 \mathrm{C}$ EX vs $2 \mathrm{~K}-1 \mathrm{C}$ SED rats $(P<0.05)$. Plasma Ang II and aldosterone were significantly higher in 2K-1C SED rats and decreased in 2K-1C EX rats to levels similar to Sham SED or Sham EX rats. Clipped kidney weights were significantly lower in both $2 \mathrm{~K}-1 \mathrm{C}$ groups, but GFR and urine flow rates were no different from right and left kidneys among the four groups. Total and fractional sodium excretion rates from the unclipped kidney of $2 \mathrm{~K}-1 \mathrm{C}$ SED rats were higher vs either Sham group $(P<0.05)$. Values in 2K-1C EX rats were similar to the Sham groups. Potassium excretion paralleled sodium excretion. These studies show that voluntary dynamic exercise in $2 \mathrm{~K}-1 \mathrm{C}$ rats decreases plasma Ang II and aldosterone, which contribute to the lower arterial pressure without deleterious effects on GFR. The effects on sodium excretion underscore the impact of pressure natriuresis despite elevated plasma Ang II and aldosterone in sedentary $2 \mathrm{~K}-1 \mathrm{C}$ rats. In contrast, potassium excretion is primarily regulated by circulating aldosterone and distal sodium delivery.
\end{abstract}

Keywords: glomerular filtration rate, Goldblatt kidney, sodium excretion, potassium excretion, sympathetic nervous system

\section{Introduction}

Regular dynamic exercise has been shown to decrease systemic arterial blood pressure, and is accompanied by diminishing efferent sympathetic nerve activity, ${ }^{1,2}$ in humans ${ }^{3,4}$ and in several, but not all, models of hypertension in rats..$^{5-8}$ In spontaneously hypertensive rats (SHR), daily volitional exercise decreases blood pressure and plasma renin activity, whereas results in stroke-prone SHR have been less consistent. ${ }^{5,7}$ In Dahl salt-sensitive rats, a model of kidney-mediated hypertension, regular dynamic exercise does not reduce systolic pressure despite decreasing the response to ganglionic blockade consistent with diminished sympathetic outflow. ${ }^{6}$ In two-kidney, one-clip $(2 \mathrm{~K}-1 \mathrm{C})$ hypertensive rats, the type of dynamic exercise influences the hemodynamic
Department of Internal Medicine, Wayne State University School of Medicine, 4160 John R Street, \#908, Detroit, MI 4820I, USA

$\mathrm{Tel}+\mathrm{I} 3137457 \mid 45$

Fax + I $313745804 \mid$

Email nrossi@wayne.edu 
response. Although neither treadmill exercise ${ }^{9,10}$ nor forced swimming exercise ${ }^{10,11}$ decreases blood pressure, voluntary wheel running exercise significantly decreases systemic arterial pressure, lowers plasma angiotensin II (Ang II) levels, and modulates renal sympathetic nerve activity in this model. ${ }^{8}$ The effect of exercise on both blood pressure and sympathetic activity is dependent on the severity of $2 \mathrm{~K}-1 \mathrm{C}$ hypertension, with reductions occurring in moderate but not severe hypertension. ${ }^{8,10}$

Improvement in structural parameters, such as collagen deposition and inflammatory infiltration, have been reported in the kidneys of exercised $2 \mathrm{~K}-1 \mathrm{C}$ rats, ${ }^{11,12}$ but scant information exists about the effects of exercise on parameters of renal function in this model. Notably, efferent renal sympathetic nerves influence three major renal functions: renal blood flow, renin secretion, and renal tubular sodium reabsorption. ${ }^{13}$ Evidence from studies with renal denervation indicate that efferent renal sympathetic nerve activity exerts little or no influence on effective renal blood flow rate in either normal ${ }^{14}$ or hypertensive rats. ${ }^{15,16}$ In contrast, renal sympathetic nerve activation increases renin secretion leading to activation of the renin-angiotensin-aldosterone cascade. ${ }^{17}$ Ang II and aldosterone actions on renal tubules are known to increase renal tubular sodium reabsorption. Moreover, direct action of norepinephrine released from nerve endings onto adrenergic receptors on the tubules enhances renal sodium reabsorption independent of changes in glomerular filtration rate (GFR). ${ }^{18,19}$ Renal denervation of the clipped kidney ${ }^{20}$ and regular dynamic exercise in $2 \mathrm{~K}-1 \mathrm{C}$ rats, ${ }^{8}$ by virtue of their sympathoinhibitory influence, significantly decrease plasma Ang II levels in $2 \mathrm{~K}-1 \mathrm{C}$ hypertensive rats. The combined decrease in direct sympathetic vasomotor tone and diminished Ang II actions on the vasculature should contribute to a decrease in systemic arterial pressure. The decline in plasma Ang II with exercise along with the expected parallel changes in aldosterone should attenuate renal tubular sodium reabsorption and further contribute to the reduction in arterial pressure.

The focus of studies on exercise and exercise training in $2 \mathrm{~K}-1 \mathrm{C}$ rats in the past has been on the impact on arterial pressure and neurocardiac function. ${ }^{8,9,11}$ Changes in renal histology have been reported, ${ }^{11}$ but direct effects on glomerular filtration and urinary excretion have not been studied. Importantly, arterial pressure in $2 \mathrm{~K}-1 \mathrm{C}$ rats is lower after only 6 weeks of exercise but the impact on renal sympathetic nerve activity is observed only after 12 weeks of voluntary wheel exercise. ${ }^{8}$ This suggests that mechanisms underlying the arterial pressure-lowering effects of regular exercise may differ over time. Thus, it is reasonable to assess the contribution that decreased renal sympathetic inputs on the renin-angiotensin-aldosterone system and tubular sodium reabsorption may exert at the 12-week time point.

Given the foregoing observations, we hypothesize that the decrease in arterial pressure seen with 12 weeks of daily wheel running exercise by moderately hypertensive $2 \mathrm{~K}-1 \mathrm{C}$ rats is due, at least in part, to decreases in plasma Ang II and aldosterone concentrations and normalization of urinary sodium and potassium excretion independent of changes in GFR.

\section{Methods}

Male Sprague Dawley rats (Harlan Sprague Dawley, Indianapolis, IN, USA) used in all the studies were housed under controlled conditions $\left(21^{\circ} \mathrm{C}-23^{\circ} \mathrm{C}\right.$; lights on, 07:00-19:00 hours) and were permitted free access to water and standard rat chow. The rats were cared for in accordance with the principles of the National Institutes of Health Guide for the Care and Use of Laboratory Animals. All protocols were approved by the Wayne State University Institutional Animal Care and Use Committee.

\section{Renal artery clipping and telemetry transmitter placement}

Five-week-old rats were anesthetized with combined ketamine $80 \mathrm{mg} / \mathrm{kg}$ and xylazine $5 \mathrm{mg} / \mathrm{kg}$ intraperitoneally (ip). A silver clip $(0.2 \mathrm{~mm})$ was surgically placed around the right renal artery (2K-1C group) via a flank incision as we have previously reported..$^{8,20,21}$ Rats in the sham-clipped (Sham) groups underwent identical surgery but were not clipped. The flank incision was closed. A ventral midline incision was made in the neck and a radiotelemetry transmitter (TA11PA-C40; Data Sciences Intl) was implanted into each rat by exposing the left carotid artery, temporarily occluding the proximal end, and inserting the gel-filled catheter attached to the transmitter device into the artery with a 21 -gauge needle. The catheter was advanced into the aorta and secured with medical adhesive and the transmitter was tunneled subcutaneously and sutured to the muscles between the scapulae. The incision was closed with surgical staples. At the end of surgery, each rat received butorphanol tartrate $0.075 \mathrm{mg}$ subcutaneously for analgesia. Each rat was returned to its individual home cage with its own telemetry receiver. Telemetric-derived hemodynamic data were recorded at the end of the protocols.

\section{Exercise and sedentary protocols}

Five days after surgery, Sham or $2 \mathrm{~K}-1 \mathrm{C}$ rats were further assigned randomly to either sedentary (SED) or exercise 
(EX) groups resulting in four groups: Sham SED, Sham EX, 2K-1C SED, and 2K-1C EX. Roughly, 15\% additional rats were placed into each EX group to account for rats that would fail to exercise within 2 SDs of the overall mean distance and would require a priori removal from the protocol. SED rats were left in their individual home cages for the duration of the experiment. EX rats were placed in cages identical to the SED rats but with access to running wheels $(1.1 \mathrm{~m}$ per revolution) equipped with an optical sensor to detect and record total revolutions (Lafayette Instruments). Running distances were averaged over 24-hour periods for each animal. Rats were allowed free access to the wheels $24 \mathrm{~h} /$ day, 7 days/week for 12 weeks. Any rat running $<1000 \mathrm{~m} /$ day for 2 weeks was excluded from analysis a priori. To avoid acute exercise-induced effects, the running wheel was closed off to the EX rats for 24 hours before undergoing further testing.

\section{Protocol I: plasma Ang II and aldosterone measurements}

After the sedentary and exercise protocols were completed, the running wheels were closed off to the exercise groups for 24 hours. Mean arterial pressure (MAP) and heart rate were assessed by telemetry in the home cage over the next 24 hours. Then, a random subset of rats from each of the four groups (Sham SED, Sham EX, 2K-1C SED, and 2K-1C EX) was chosen to assess plasma Ang II and aldosterone. This approach sought to minimize the impact of surgical stress, fluid infusion, and blood drawing from the clearance experiments that may have independently influenced the hormone levels. Each rat was anesthetized with pentobarbital sodium ( $50 \mathrm{mg} / \mathrm{kg}$ ip). The aorta was exposed via midline abdominal incision. A ligature was placed on distal aorta just above the bifurcation of the iliac arteries. A suture was loosely placed around the proximal aorta between the superior mesenteric and renal arteries. The aorta was rapidly cannulated and $2 \mathrm{~mL}$ of blood was collected for subsequent analysis of Ang II and aldosterone. Then, the rat was euthanized and the kidneys were harvested and weighed.

\section{Protocol 2: renal clearance and excretion}

A separate set of rats from each of the four groups underwent assessment of GFR and urinary sodium and potassium excretion rates using standard clearance methodology we have previously reported..$^{22}$ As in Protocol 1, the running wheels were closed off to the exercised groups for 24 hours, and then hemodynamic data were recorded during the next 24 hours.

Each rat was anesthetized with pentobarbital sodium (50 $\mathrm{mg} / \mathrm{kg}$ ip). A 23-gauge polyethylene catheter was inserted into the right femoral artery for blood pressure monitoring and for blood sampling and into the right femoral vein for infusion. Arterial pressure was monitored continuously during the clearance periods by connecting the arterial catheter to a pressure transducer (Gould P23 XL) coupled to an amplifier (Digi0Med BPA-200). The heart rate was derived from the beat-to-beat arterial pressure pulse and averaged over 1 -second intervals using custom programmed data acquisition software (Dasylab; Biotech Products). To determine GFR, a bolus of $2.0 \mathrm{~mL} / \mathrm{kg}$ body weight of $8 \mathrm{mg} / \mathrm{mL}$ fluorescein isothiocyanate (FITC)-inulin (Sigma-Aldrich Co.) in $0.9 \%$ saline was infused followed by continuous infusion of $4 \mathrm{mg} /$ $\mathrm{mL}$ at $0.055 \mathrm{~mL} / \mathrm{min}$ to maintain a steady-state plasma inulin concentration and to account for fluid loss. ${ }^{23}$ The right and left ureters were cannulated with 27-gauge polyethylene catheters for urine sampling from each kidney. After a 30-minute stabilization period, urine was collected for two consecutive 15 -minute time periods, and an arterial blood sample $(0.2 \mathrm{~mL})$ was collected from the femoral arterial catheter at the midpoint of each collection to assess plasma inulin, sodium, and potassium concentrations. Rats were then euthanized with an overdose of sodium pentobarbital ( $200 \mathrm{mg} / \mathrm{kg}$ intravenously). The kidneys were collected and weighed.

\section{Analytical measurements and calculations}

Plasma and urinary FITC-inulin concentrations were quantitated using a Synergy H1 microplate reader (Biotek Instruments) at Ex/Em 485/535. Plasma and urinary sodium and potassium concentrations were determined with flame photometry with internal lithium standardization (Cole Parmer Instruments). Urine volume was assessed gravimetrically.

GFR as well as urinary excretion rates of sodium and potassium and fractional excretion of sodium and potassium were assessed using standard formulae. Inulin clearance values were expressed after correction for body weight $(\mathrm{mL} /$ $\mathrm{min} / \mathrm{kg} \mathrm{BW})$ as well as for kidney weight $(\mathrm{mL} / \mathrm{min} / \mathrm{g} \mathrm{KW})$.

Plasma samples for Ang II were processed as described previously ${ }^{24}$ with minimal modification. Briefly, $1 \mathrm{~mL}$ of plasma was extracted twice with $90 \%$ methanol. The extracts were combined, taken to dryness under nitrogen, and stored overnight at $-20^{\circ} \mathrm{C}$. The extracts were reconstituted in $0.5 \mathrm{~mL}$ assay buffer consisting of $50 \mathrm{mM}$ Na phosphate, $1 \mathrm{mM}$ ethylenediaminetetraacetic acid, $0.25 \mathrm{mM}$ thimerosal, and $0.25 \%$ peptidase-free human serum albumin. All samples were assayed in duplicate using ${ }^{125}$ I-labeled Ang II (Perkin-Elmer, Billerica, MA, USA) as the tracer and anti-Ang II antibody (Peninsula Laboratories, San Carlos, CA, USA) at final dilution of 1:660,000. Nonspecific binding was $2.1 \%$, the lower 
limit of detection was $0.5 \mathrm{fmol} / \mathrm{tube}$, and $50 \%$ binding was $15.2 \mathrm{fmol} /$ tube. Plasma aldosterone was assessed directly on plasma with ${ }^{125} \mathrm{I}$-aldosterone as the tracer in the solid phase Coat-a-Count radioimmunoassay system (Siemens).

\section{Statistical analysis}

Values are presented as mean \pm SE. All comparisons among groups were made by one-way analysis of variance with the Tukey-Kramer post hoc analysis for multiple comparisons. Comparisons within the same group of rats were analyzed by paired $t$-test. A $P$-value $<0.05$ was taken as significant.

\section{Results}

Running distances by the Sham EX and 2K-1C EX rats over the 12 weeks were comparable for Protocol 1 (Figure 1, upper panel) and Protocol 2 (Figure 1, lower panel). The 2K-1C rats in Protocol 2 tended to run greater distances early in the experiment but distances became similar after week 5 . One $2 \mathrm{~K}-1 \mathrm{C}$ rat in Protocol 2 consistently ran $<800 \mathrm{~m} /$ day for 3 weeks and was excluded from the study by design.
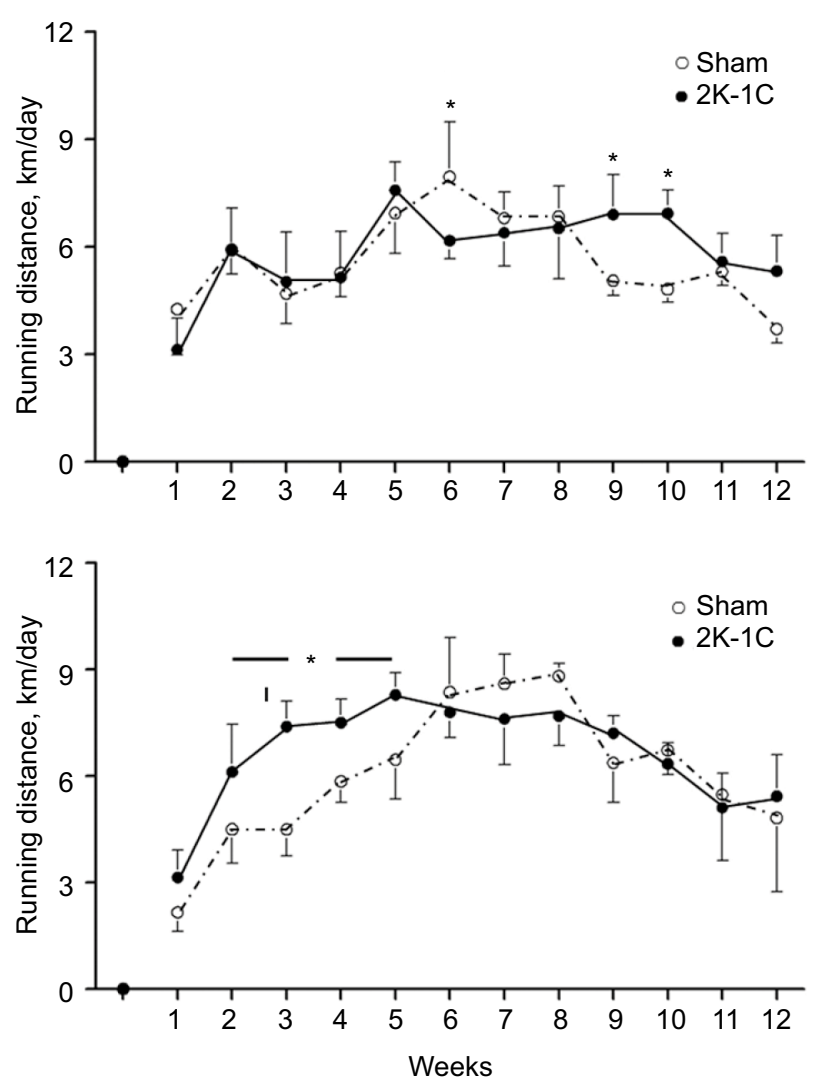

Figure I Running distances in the rats in Protocols I and 2.

Notes: Average running distances over the course of 12 weeks of voluntary wheel running for Sham EX $(n=12)$ and 2K-IC EX $(n=7)$ rats in Table I (Protocol I; upper panel) and for Sham EX ( $n=7)$ and 2K-IC EX ( $n=7)$ rats in Table 2 (Protocol 2; lower panel). Values are mean $\pm S E ;{ }^{*} P<0.05$ vs Sham.

Abbreviations: 2K-IC, two-kidney, one-clip; Sham, sham clipped; EX, exercise.

\section{Protocol I}

Parameters for rats in Protocol 1 are provided in Table 1. Body weights of Sham and $2 \mathrm{~K}-1 \mathrm{C}$ rats were similar at the beginning of Protocol 1. At the end of the 12 weeks, body weights of both EX groups were similar to each other but were significantly less than their respective SED groups. This occurred despite equivalent caloric intake (average $\sim 14 \mathrm{~g}$ chow/day/rat in all groups). Kidney weights of the right (clipped) kidney were significantly lower than those of the left kidney in both $2 \mathrm{~K}-1 \mathrm{C}$ groups. Diurnal variation during the brief 24-hour period of telemetric data collection appeared to be preserved and the differences among the groups during daytime and nighttime MAP were similar (data not shown). MAP assessed by telemetry and averaged over the entire 24-hour period was significantly lower in the 2K-1C EX group compared with the 2K-1C SED group. Exercise training did not result in a difference in MAP in the Sham EX rats but this group displayed a significant bradycardic effect compared with Sham SED. The 2K-1C SED rats exhibited significantly elevated levels of plasma Ang II and aldosterone compared with either Sham SED or Sham EX rats. Plasma Ang II and aldosterone in the 2K-1C EX rats decreased significantly compared with $2 \mathrm{~K}-1 \mathrm{C}$ SED rats and were not significantly different from those in either of the Sham groups.

\section{Protocol 2}

Average body weights at the end of 12 weeks in Protocol 2 did not differ significantly among the four groups (Table 2 ). Initial weights were also similar among the groups (data not shown). MAP assessed by telemetry was higher in the $2 \mathrm{~K}-1 \mathrm{C}$ SED group (189.5 $\pm 8.2 \mathrm{mmHg}$ ) compared with either Sham SED $(132.0 \pm 2.8 \mathrm{mmHg})$ or Sham EX $(133.9 \pm 2.7 \mathrm{mmHg})$ groups $(P<0.01)$. MAP in the $2 \mathrm{~K}-1 \mathrm{C}$ EX rats was lower in the $2 \mathrm{~K}-1 \mathrm{C}$ EX group $(162.7 \pm 6.7 \mathrm{mmHg})$ compared with the $2 \mathrm{~K}-1 \mathrm{C}$ SED rats $(P<0.05)$, but remained higher than in either of the Sham groups $(P<0.05)$. Similar to measurements in conscious rats acquired by telemetry, MAP in $2 \mathrm{~K}-1 \mathrm{C}$ SED rats after anesthesia for clearance studies was lower $(P<0.05$ vs telemetry) than in the conscious state but remained significantly higher than MAP in any of the other groups (Table 2). Notably, the decrease in MAP with anesthesia was less evident in the Sham groups and the $2 \mathrm{~K}-1 \mathrm{C}$ EX group and did not achieve significance $(\sim 1-5 \mathrm{mmHg} ; P>0.05)$.

As in Protocol 1, the Sham EX group displayed a significantly lower heart rate $(372 \pm 10 \mathrm{bpm})$ compared with the Sham SED group ( $425 \pm 11 \mathrm{bpm}, P<0.05)$. The $2 \mathrm{~K}-1 \mathrm{C}$ EX group had a lower heart rate $(384 \pm 6 \mathrm{bpm})$ compared with 2K-1C SED group (402 $\pm 10 \mathrm{bpm})$, but this did not achieve significance $(P>0.05)$. 
Table I Body weights, kidney weights, baseline resting hemodynamic parameters, and plasma Ang II and aldosterone levels in shamclipped and two-kidney, one-clip hypertensive rats after 12-week sedentary or exercise conditions (Protocol I)

\begin{tabular}{|c|c|c|c|c|c|c|c|c|c|}
\hline \multirow[t]{2}{*}{ Groups } & \multirow[t]{2}{*}{$\mathbf{N}$} & \multirow{2}{*}{$\begin{array}{l}\text { BW } \\
\text { beginning } \\
\mathrm{g}\end{array}$} & \multirow{2}{*}{$\begin{array}{l}\text { BW after } \\
12 \text { weeks } \\
\mathrm{g}\end{array}$} & \multicolumn{2}{|l|}{ KW (g) } & \multirow{2}{*}{$\begin{array}{l}\text { MAP } \\
\overline{\mathrm{mmHg}}\end{array}$} & \multirow{2}{*}{$\begin{array}{l}\text { Heart } \\
\text { rate } \\
\text { bpm }\end{array}$} & \multirow{2}{*}{$\begin{array}{l}\text { Plasma } \\
\text { Ang II } \\
\mathrm{fmol} / \mathrm{mL}\end{array}$} & \multirow{2}{*}{$\begin{array}{l}\text { Plasma } \\
\text { aldosterone } \\
\mathrm{pg} / \mathrm{mL}\end{array}$} \\
\hline & & & & Left & Right & & & & \\
\hline Sham SED & 9 & $|6| \pm 3$ & $407 \pm 10$ & $1.63 \pm 0.07$ & $1.68 \pm 0.05$ & $|33 \pm|$ & $415 \pm 12$ & $29.3 \pm 8.1$ & $577 \pm 209$ \\
\hline Sham EX & 12 & $157 \pm 3$ & $370 \pm 8^{a}$ & $1.53 \pm 0.07$ & $1.49 \pm 0.06$ & $135 \pm 1$ & $379 \pm 9^{a}$ & $47.3 \pm 7.1$ & $304 \pm 80$ \\
\hline $2 \mathrm{~K}-\mathrm{IC}$ SED & 7 & $153 \pm 3$ & $432 \pm 10$ & $1.7 I \pm 0.73$ & $1.30 \pm 0.12^{\mathrm{d}}$ & $191 \pm 4^{\mathrm{a}, \mathrm{b}}$ & $379 \pm 11$ & $187.4 \pm 43.6^{\mathrm{a}, \mathrm{b}}$ & $3.173 \pm 1.213^{\mathrm{a}, \mathrm{b}}$ \\
\hline $2 \mathrm{~K}-\mathrm{IC}$ EX & 7 & $158 \pm 6$ & $374 \pm 9$ & $I .5 \mathrm{I} \pm 0.70$ & $1.17 \pm 0.06^{\mathrm{a}, \mathrm{d}}$ & $169 \pm 2^{a-c}$ & $392 \pm 5$ & $48.9 \pm 7.5^{c}$ & $739 \pm 151^{c}$ \\
\hline
\end{tabular}

Notes: Values are mean \pm SE; right kidneys clipped. ${ }^{a} P<0.01$ vs Sham SED; ${ }^{b} P<0.01$ vs Sham EX; ${ }^{c} P<0.05$ vs $2 K-I C$ SED; ${ }^{d} P<0.05$ vs left kidney.

Abbreviations: BW, body weight; EX, exercise; KW, kidney weight; MAP, mean arterial pressure; SED, sedentary; 2K-IC, two-kidney, one-clip.

The clipped kidneys exhibited significantly lower kidney weights. GFRs did not differ among the groups. When GFR was expressed per body weight, there was no difference between left and right kidneys. When GFR was expressed per kidney weight, in light of the differences in kidney weights, the clipped kidney of the 2K-1C SED group was significantly higher than that of the nonclipped, left kidney.

Plasma sodium concentrations were similar among the groups. Plasma potassium levels were significantly lower in the $2 \mathrm{~K}-1 \mathrm{C}$ SED rats compared with values in the three other groups (Table 2). Figure 2 shows that the left unclipped kidneys in the 2K-1C SED group displayed higher sodium excretion rates than the left kidneys of either of the Sham groups. Sodium excretion by the unclipped kidney of the $2 \mathrm{~K}-1 \mathrm{C}$ EX group was significantly lower than that of the $2 \mathrm{~K}-1 \mathrm{C}$ SED group and did not differ from that of either of the Sham groups. Sodium excretion by the clipped kidneys did not differ among any of the groups. Fractional sodium excretion exhibited the same pattern among the groups (Figure 3).

Potassium excretion rates from the unclipped kidneys were higher in the $2 \mathrm{~K}-1 \mathrm{C}$ SED rats than either Sham SED or Sham EX rats (Figure 2). Potassium excretion was also higher from the unclipped kidneys in the 2K-1C SED rats compared with Sham EX rats. The $2 \mathrm{~K}-1 \mathrm{C}$ EX group displayed potassium excretion rates from both left and right kidneys that were significantly lower than the corresponding kidneys of the 2K-1C SED group. Potassium excretion from right or left kidneys did not differ from the corresponding kidneys in either of the Sham groups. Likewise, fractional excretion of potassium from the left unclipped kidney was significantly higher in the 2K-1C SED group compared with either Sham group and was significantly lower from the left kidney of the 2K-1C EX group (Figure 3).

\section{Discussion}

As the prevalence of renovascular hypertension rises, ${ }^{25}$ knowledge about the impact of regular voluntary dynamic exercise on blood pressure and renal function in this condition becomes vital. The present study reports several findings in the $2 \mathrm{~K}-1 \mathrm{C}$ rat, a model of unilateral renal artery stenosis. First, daily exercise for 12 weeks decreased arterial pressure in $2 \mathrm{~K}-1 \mathrm{C}$ hypertensive rats. Second, with daily exercise in $2 \mathrm{~K}-1 \mathrm{C}$ rats, elevated plasma Ang II and aldosterone levels returned to levels not different from those observed in the sham-clipped rats. Third, GFR did not differ between sedentary sham and $2 \mathrm{~K}-1 \mathrm{C}$ groups and, further, was not altered by exercise in either group. Fourth, both absolute and fractional urinary sodium and potassium excretion values from the nonclipped kidneys of sedentary $2 \mathrm{~K}-1 \mathrm{C}$ rats was significantly elevated compared with that of sham-clipped rats. Notably, urinary sodium and potassium excretion by the $2 \mathrm{~K}-1 \mathrm{C}$ exercised rats was not different from that of sham-clipped normotensive rats.

\section{Hemodynamic response to exercise training}

Our data indicate that voluntary wheel running exercise in $2 \mathrm{~K}-1 \mathrm{C}$ rats decreased arterial pressure when measured by telemetry in conscious freely moving rats or by direct arterial pressure recording in pentobarbital-anesthetized rats. The arterial pressure-lowering effect of exercise training was most evident in the hypertensive $2 \mathrm{~K}-1 \mathrm{C}$ group rather than the sham-clipped normotensive rats. The blood pressure-lowering effect of exercise training in $2 \mathrm{~K}-1 \mathrm{C}$ hypertension has been observed by some investigators ${ }^{8,26}$ but not others. ${ }^{9-11}$ These discrepancies may be related to the type of exercise regimen, the technique used for assessing blood pressure, or the presence, type, or depth of anesthesia. For example, an 8- to 12-week forced treadmill regimen did not decrease arterial pressure. ${ }^{9,10}$ Forced swimming exercise over a 4-week period reduced arterial pressure to an extent similar to that seen in the present study. ${ }^{11,26}$ However, Maia et al ${ }^{11}$ showed that additional weeks of forced swimming resulted in no difference between exercise and sedentary $2 \mathrm{~K}-1 \mathrm{C}$ groups. The 


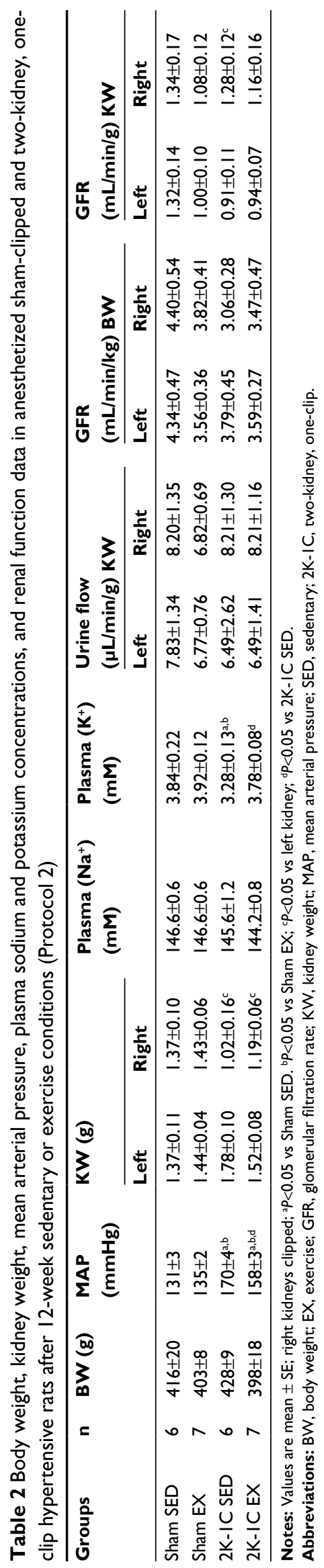

disruption of circadian rhythms may also have reduced the impact of forced treadmill or swimming exercise on arterial pressure, ${ }^{27}$ whereas voluntary wheel exercise is performed during the normal waking cycle of the rat. The use of tail cuff measurements in some of these earlier studies may have obscured differences in blood pressure because of concurrent restraint stress ${ }^{28}$ which exerts a disproportionate influence on higher arterial pressures. ${ }^{29}$ Notably, sodium pentobarbital anesthesia may affect blood pressure in hypertensive rats to a greater extent than in normotensive animals. ${ }^{30,31}$ Consistent with these earlier studies, the hypertensive sedentary $2 \mathrm{~K}-1 \mathrm{C}$ displayed a significant difference in MAP between conscious and anesthetized states, whereas this was not observed in the sham-clipped normotensive groups. Notably, anesthesia had an attenuated effect to lower MAP in the exercised $2 \mathrm{~K}-1 \mathrm{C}$ rats (Table 2). Nonetheless, the impact of regular exercise on MAP was preserved when comparing $2 \mathrm{~K}-1 \mathrm{C}$ sedentary vs exercised rats. Our studies confirm earlier findings that voluntary wheel running exercise decreases arterial pressure in $2 \mathrm{~K}-1 \mathrm{C}$ rats under both conscious and anesthetized conditions.

We observed a significant bradycardic response to exercise training in the sham-clipped but not the $2 \mathrm{~K}-1 \mathrm{C}$ rats in both protocols. Although $2 \mathrm{~K}-1 \mathrm{C}$ rats clearly exhibit increased sympathetic nerve activity, ${ }^{20} 2 \mathrm{~K}-1 \mathrm{C}$ rats $^{32,33}$ and mice $^{34}$ display decreased parasympathetic tone. The same has been suggested in human renovascular hypertension. ${ }^{35}$ The lack of effect of voluntary wheel running exercise on heart rate has also been reported in Dahl salt-sensitive rats. ${ }^{6}$ While daily spontaneous wheel running decreases renal sympathetic activity in the $2 \mathrm{~K}-1 \mathrm{C}$ model, ${ }^{8}$ it is possible that exercise-induced augmentation of parasympathetic control of heart rate may be less effective in $2 \mathrm{~K}-1 \mathrm{C}$ than sham-clipped normotensive rats or that renal sympathetic tone may decrease to a greater extent than cardiac sympathetic activity. ${ }^{36} \mathrm{It}$ should be noted that the study was not powered to evaluate changes in heart rate, which tends to have greater variability.

\section{Plasma Ang II and aldosterone levels}

Plasma Ang II and aldosterone levels in both groups of shamclipped rats and sedentary $2 \mathrm{~K}-1 \mathrm{C}$ rats were elevated similar to values reported previously. ${ }^{24,37,38}$ Daily exercise clearly decreased plasma Ang II and aldosterone levels to concentrations similar to those in sham-clipped animals. Taken together with evidence indicating that daily exercise attenuates renal sympathetic nerve activity ${ }^{2,8}$ and that sympathetic inputs to the kidney regulate activity of the renin-angiotensin-aldosterone system, ${ }^{17}$ the decrease in plasma Ang II and aldosterone levels in $2 \mathrm{~K}-1 \mathrm{C}$ exercised rats strongly supports the concept 

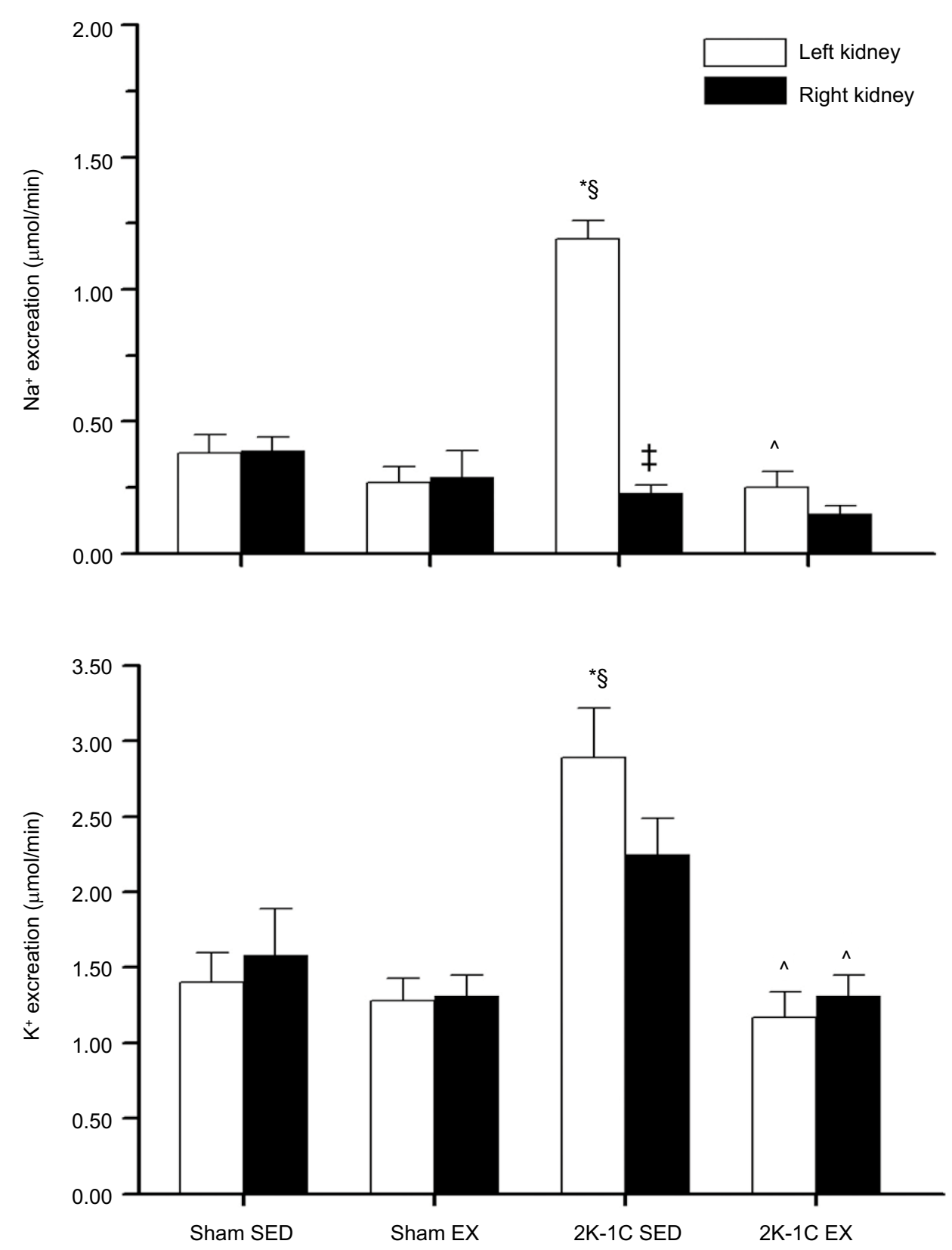

Figure 2 Total urinary sodium and potassium excretion rates.

Notes: Urinary excretion rates for sodium (upper panel) and potassium (lower panel) for left and right kidneys in the four groups. Values are mean \pm SE; $\mathrm{n}$ as in Table 2. $* P<0.001$ vs Sham SED; ${ }^{\$} P<0.05$ vs Sham EX; ${ }^{*} P<0.00$ I vs left kidney; and ${ }^{\wedge} P<0.001$ vs $2 \mathrm{~K}-\mathrm{IC}$ SED.

Abbreviations: 2K-IC SED, two-kidney, one-clip sedentary; 2K-IC EX, two-kidney, one-clip exercise; Sham EX, sham-clipped exercise; Sham SED, sham-clipped sedentary.

that sympathoinhibition due to exercise can attenuate activation of the renin-angiotensin-aldosterone system in $2 \mathrm{~K}-1 \mathrm{C}$ hypertension. As circulating Ang II and aldosterone promote renal sympathoexcitation, ${ }^{39,40}$ the decline in both of these hormones may, in turn, further contribute to the sympathoinhibitory effect of regular exercise and lowering of arterial pressure in this model.

\section{Glomerular filtration rate}

In the present study, GFR did not differ significantly between sedentary sham-clipped and $2 \mathrm{~K}-1 \mathrm{C}$ groups. This has been observed in some, ${ }^{41-43}$ but not all, ${ }^{44}$ earlier studies of the $2 \mathrm{~K}-1 \mathrm{C}$ model and may have depended on the size of the clip, the age of the rat at the time of clipping, and the time after clipping at which renal function was assessed.

In addition, GFR remained unchanged in exercised 2K-1C rats despite lowering arterial pressure and Ang II. Our previous studies have shown that voluntary wheel running exercise inhibits renal sympathetic nerve activity. ${ }^{8}$ A possible explanation is that the combined effects of sympathoinhibition and lower Ang II result in a greater decrease in afferent vs efferent arteriolar resistance thereby 

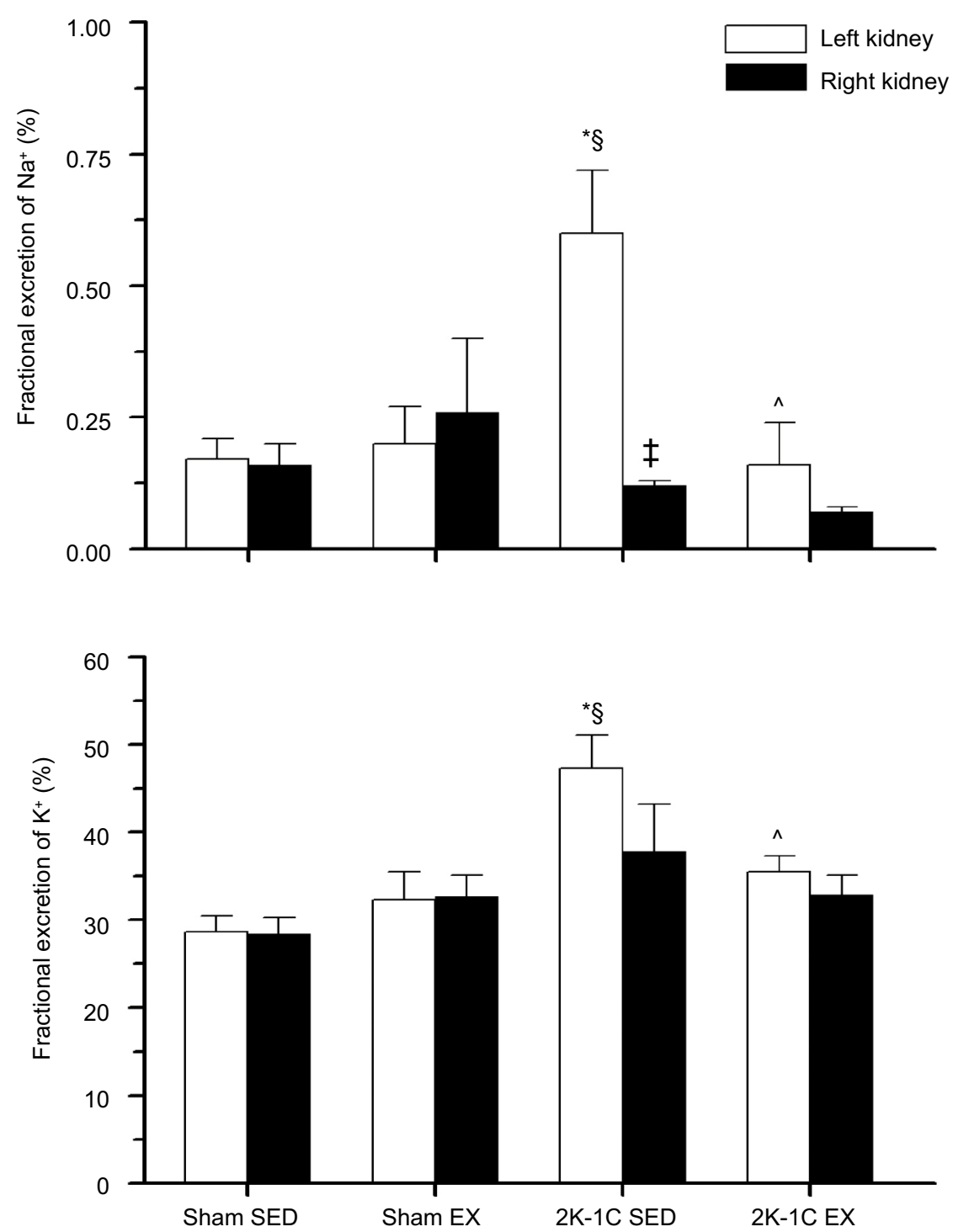

Figure 3 Fractional excretion of sodium and potassium.

Notes: Fractional excretion of sodium (upper panel) and potassium (lower panel) for left and right kidneys in the four groups. Values are mean $\pm \mathrm{SE} ; \mathrm{n}$ as in Table 2 . $* P<0.0 \mathrm{I}$ vs Sham SED; ${ }^{\circledR P}<0.05$ vs Sham EX; ${ }^{\ddagger} P<0.00$ I vs left kidney; and ${ }^{\wedge} P<0.05$ vs $2 \mathrm{~K}-$ IC SED.

Abbreviations: 2K-IC EX, two-kidney, one-clip exercise; 2K-IC SED, two-kidney, one-clip sedentary; Sham EX, sham-clipped exercise; Sham SED, sham-clipped sedentary.

maintaining intraglomerular pressure and GFR. These data are consistent with existing studies demonstrating that pharmacologic inhibition of Ang II actions alone does not decrease glomerular filtration. ${ }^{42,45,46}$ In addition, glomerular filtrate rate remains unchanged when Ang II is blocked after acute renal denervation in $2 \mathrm{~K}-1 \mathrm{C}$ rats. ${ }^{43}$ Thus, regular dynamic exercise does not exert a detrimental effect on GFR in moderately hypertensive $2 \mathrm{~K}-1 \mathrm{C}$ rats. The small but significant increase in GFR from the clipped kidney of sedentary $2 \mathrm{~K}-1 \mathrm{C}$ when expressed per kidney weight may be due to a more complex interplay of the sympathetic nervous system and both systemic and intrarenal renin-angiotensin systems. While the elevated systemic
Ang II levels may serve to preserve GFR via preferential efferent arteriolar vasoconstriction, intrarenal Ang II has been known to modulate tubuloglomerular feedback favoring afferent vasoconstriction. ${ }^{47}$ Intrarenal Ang II was high in the clipped kidney 1 week after renal artery clipping; however, after 9 weeks, renal cortical Ang II decline levels were no different from those in sham-clipped rats. ${ }^{20}$ Thus, the timing after clipping may also contribute substantially to the impact on GFR. It is plausible that efferent arteriolar vasoconstriction in response to systemic Ang II, which is threefold higher in the sedentary $2 \mathrm{~K}-1 \mathrm{C}$ group, serves to maintain GFR at 12 weeks. Studies designed to assess these complex interactions would be required. 


\section{Excretory function}

The higher urinary sodium excretion rate by the unclipped kidney but not the clipped kidney in the sedentary $2 \mathrm{~K}-1 \mathrm{C}$ group reflects the substantial influence that renal perfusion pressure exerts via pressure natriuresis. ${ }^{48}$ Our findings are consistent with studies showing that acutely elevated levels of Ang II are antinatriuretic when renal perfusion pressure is prevented from increasing but natriuretic when renal perfusion pressure is permitted to rise. ${ }^{49} \mathrm{~A}$ similar excretory pattern has been observed in $2 \mathrm{~K}-1 \mathrm{C}$ rats when renal perfusion pressure is controlled.$^{41}$ This would not, however, fully explain how urinary sodium excretion was virtually identical to that in the sham-clipped groups although arterial pressure was still significantly elevated in the exercise-trained $2 \mathrm{~K}-1 \mathrm{C}$ group. Thus, only a component of the decrease in sodium excretion can be attributed to a decrease in renal perfusion pressure.

Together with the known inhibition of renal sympathetic activity, ${ }^{2,8}$ the observed decline in plasma Ang II would have predicted an increase in urinary sodium excretion with regular exercise in the $2 \mathrm{~K}-1 \mathrm{C}$ group. ${ }^{43}$ Consistent with our findings, renal sympathetic innervation is paradoxically permissive for sodium excretion in response to exogenous Ang II in the canine split bladder model. ${ }^{50}$ Despite a decrease of plasma Ang II to values similar to sham-clipped animals, denervation of the clipped kidney results in a profound decrease in renal sympathetic nerve activity but no change in intrarenal Ang II to the unclipped kidney. ${ }^{20}$ Although renal tissue Ang II was not measured in the current study, it is possible that persistent tubular effects by intrarenal Ang II contributed to the decrease in sodium excretion by the exercise-trained $2 \mathrm{~K}-1 \mathrm{C}$ rats. ${ }^{45}$ Furthermore, as these short-term clearance experiments were performed at the end of the 12-week exercise regimen at a time when the animals were likely in electrolyte balance, we cannot exclude the possibility that a greater or lesser amount of sodium was excreted earlier in the course by the exercised $2 \mathrm{~K}-1 \mathrm{C}$ rats. ${ }^{51,52}$

In contrast to the results with sodium excretion, the impact on urinary potassium excretion appears more straightforward. The elevated plasma aldosterone levels in the sedentary $2 \mathrm{~K}-1 \mathrm{C}$ rats contributed to the greater urinary potassium excretion by the unclipped kidney despite lower plasma potassium levels. Increased sodium delivery to the distal nephron segments also potentially increased potassium secretion $^{53}$ despite a counter-regulatory effect by Ang II. ${ }^{54}$ By decreasing aldosterone, daily exercise attenuated urinary potassium losses and restored plasma potassium levels. In addition, exercise-induced sympathoinhibition may have further contributed to the lower urinary potassium excretion in the exercised $2 \mathrm{~K}-1 \mathrm{C}$ rats ${ }^{43}$ thereby further supporting the dual action of diminished activation of the renin-angiotensin-aldosterone system and sympathetic nervous system.

\section{Summary and perspectives}

In summary, these studies support the concept that voluntary dynamic exercise lowers systemic arterial pressure and decreases plasma Ang II and aldosterone levels in the $2 \mathrm{~K}-1 \mathrm{C}$ model of renovascular hypertension without deleterious effects on GFR. The differences among the groups in sodium excretion underscore the impact of pressure natriuresis despite elevated plasma Ang II and aldosterone in the sedentary $2 \mathrm{~K}-1 \mathrm{C}$ rats. In contrast, potassium excretion appears to be primarily regulated by ambient aldosterone levels and distal sodium delivery. Importantly, atherosclerotic renal artery stenosis is increasing in prevalence, particularly in high-risk populations, and carries a high risk of cardiovascular morbidity and mortality. ${ }^{25}$ In addition to the beneficial effects on arterial pressure, regular dynamic exercise may be of benefit via diminished circulating Ang II and aldosterone, both of which contribute to deleterious cardiac and vascular remodeling. ${ }^{55,56}$ Existing studies in humans evaluated renal function during exercise and suggested a detrimental effect in renovascular hypertension. ${ }^{57}$ To our knowledge, followup investigations of the impact of regular exercise on renal function have not occurred. Our findings strongly support the possibility of beneficial long-term effects and need for further studies on the cardiovascular and renal impact of regular dynamic exercise in humans with renovascular hypertension.

\section{Acknowledgment}

This study was supported by a Merit Award from the Department of Veterans Affairs to NFR.

\section{Disclosure}

The authors report no conflicts of interest in this work.

\section{References}

1. Brum PC, Da Silva GJ, Moreira ED, Ida F, Negrao CE, Krieger EM Exercise training increases baroreceptor gain sensitivity in normal and hypertensive rats. Hypertension. 2000;36(6):1018-1022.

2. Negrao CE, Irigoyen MC, Moreira ED, Brum PC, Freire PM, Krieger EM. Effect of exercise training on RSNA, baroreflex control, and blood pressure responsiveness. Am J Physiol. 1993;265 (2 Pt 2):R365-R370.

3. Boman K, Gerdts E, Wachtell K, et al. Exercise and cardiovascular outcomes in hypertensive patients in relation to structure and function of left ventricular hypertrophy: the LIFE study. Eur J Cardiovasc Prev Rehabil. 2009;16(2):242-248.

4. Kokkinos P, Pittaras A, Manolis A, et al. Exercise capacity and 24-h blood pressure in prehypertensive men and women. Am J Hypertens. 2006;19(3):251-258. 
5. Overton JM, Tipton CM, Matthes RD, Leininger JR. Voluntary exercise and its effects on young SHR and stroke-prone hypertensive rats. J Appl Physiol (1985). 1986;61(1):318-324.

6. Overton JM, VanNess JM, Takata HJ. Effects of chronic exercise on blood pressure in Dahl salt-sensitive rats. Am J Hypertens. 1998;11(1 Pt 1):73-80.

7. Noguchi T, Sasaki Y, Seki J, Giddings JC, Yamamoto J. Effects of voluntary exercise and L-arginine on thrombogenesis and microcirculation in stroke-prone spontaneously hypertensive rats. Clin Exp Pharmacol Physiol. 1999;26(4):330-335.

8. Rossi NF, Chen H, Maliszewska-Scislo M. Paraventricular nucleus control of blood pressure in two-kidney, one-clip rats: effects of exercise training and resting blood pressure. Am J Physiol Regul Integr Comp Physiol. 2013;305(11):R1390-R1400.

9. Boissiere J, Eder V, Machet MC, Courteix D, Bonnet P. Moderate exercise training does not worsen left ventricle remodeling and function in untreated severe hypertensive rats. J Appl Physiol (1985). 2008;104(2):321-327.

10. Marcus KD, Tipton CM. Exercise training and its effects with renal hypertensive rats. J Appl Physiol (1985). 1985;59(5):1410-1415.

11. Maia RC, Sousa LE, Santos RA, et al. Time-course effects of aerobic exercise training on cardiovascular and renal parameters in 2K1C renovascular hypertensive rats. Braz J Med Biol Res. 2015;48(11):1010-1022.

12. Soares ER, Lima WG, Machado RP, et al. Cardiac and renal effects induced by different exercise workloads in renovascular hypertensive rats. Braz J Med Biol Res. 2011;44(6):573-582.

13. DiBona GF, Kopp UC. Neural control of renal function. Physiol Rev. 1997;77(1):75-197.

14. Bello-Reuss E, Colindres RE, Pastoriza-Munoz E, Mueller RA, Gottschalk CW. Effects of acute unilateral renal denervation in the rat. J Clin Invest. 1975;56(1):208-217.

15. Koepke JP, Jones S, DiBona GF. Renal nerve activity and renal function during environmental stress in DOCA-NaCl rats. Am J Physiol. 1986;251(2 Pt 2):R289-R294.

16. Krueger AD, Lee JY, Yang PC, Papaioannou SE, Walsh GM. Selective vasodilation produced by renal denervation in adult spontaneously hypertensive rats. Hypertension. 1986;8(5):372-378.

17. Kopp UC, DiBona GF. Interaction between neural and nonneural mechanisms controlling renin secretion rate. Am J Physiol. 1984;246(5 Pt 2):F620-F626.

18. Barajas L, Powers K. Monoaminergic innervation of the rat kidney: a quantitative study. Am J Physiol. 1990;259(3 Pt 2):F503-F511.

19. Bencsath P, Szenasi G, Takacs L. Water and electrolyte transport in Henle's loop and distal tubule after renal sympathectomy in the rat. $\mathrm{Am}$ J Physiol. 1985;249(2 Pt 2):F308-F314.

20. Rossi NF, Pajewski R, Chen H, Littrup PJ, Maliszewska-Scislo M. Hemodynamic and neural responses to renal denervation of the nerve to the clipped kidney by cryoablation in two-kidney, one-clip hypertensive rats. Am J Physiol Regul Integr Comp Physiol. 2016;310(2): R197-R208.

21. Rossi NF, Maliszewska-Scislo M, Chen H, et al. Neuronal nitric oxide synthase within paraventricular nucleus: blood pressure and baroreflex in two-kidney, one-clip hypertensive rats. Exp Physiol. 2010;95(8):845-857.

22. Augustyniak RA, Singh K, Zeldes D, Singh M, Rossi NF. Maternal protein restriction leads to hyperresponsiveness to stress and saltsensitive hypertension in male offspring. Am J Physiol Regul Integr Comp Physiol. 2010;298(5):R1375-R1382.

23. Churchill PC, Rossi NF, Churchill MC, Bidani AK, McDonald FD. Acute cyclosporine-induced renal vasoconstriction: lack of effect of theophylline. Am J Physiol. 1990;258(1 Pt 2):F41-F45.

24. Maliszewska-Scislo M, Chen H, Augustyniak RA, Seth D, Rossi NF. Subfornical organ differentially modulates baroreflex function in normotensive and two-kidney, one-clip hypertensive rats. Am J Physiol Regul Integr Comp Physiol. 2008;295(3):R741-R750.
25. de Mast Q, Beutler JJ. The prevalence of atherosclerotic renal artery stenosis in risk groups: a systematic literature review. $J$ Hypertens. 2009;27(7):1333-1340.

26. Rodrigues MC, Campagnole-Santos MJ, Machado RP, et al. Evidence for a role of AT(2) receptors at the CVLM in the cardiovascular changes induced by low-intensity physical activity in renovascular hypertensive rats. Peptides. 2007;28(7):1375-1382.

27. van den Buuse M. Circadian rhythms of blood pressure and heart rate in conscious rats: effects of light cycle shift and timed feeding. Physiol Behav. 1999;68(1-2):9-15.

28. Grundt A, Grundt C, Gorbey S, Thomas MA, Lemmer B. Straindependent differences of restraint stress-induced hypertension in WKY and SHR. Physiol Behav. 2009;97(3-4):341-346.

29. Feng M, Whitesall S, Zhang Y, Beibel M, D’Alecy L, DiPetrillo K. Validation of volume-pressure recording tail-cuff blood pressure measurements. Am J Hypertens. 2008;21(12):1288-1291.

30. Bencze M, Behuliak M, Zicha J. The impact of four different classes of anesthetics on the mechanisms of blood pressure regulation in normotensive and spontaneously hypertensive rats. Physiol Res. 2013;62(5):471-478.

31. Walker LA, Gellai M, Valtin H. Renal response to pentobarbital anesthesia in rats: effect of interrupting the renin-angiotensin system. J Pharmacol Exp Ther. 1986;236(3):721-728.

32. Chen JK, Zhao T, Ni M, Li DJ, Tao X, Shen FM. Downregulation of alpha7 nicotinic acetylcholine receptor in two-kidney one-clip hypertensive rats. BMC Cardiovasc Disord. 2012;12:38.

33. Cabral AM, Vasquez EC. Time course of cardiac sympathetic and vagal tone changes in renovascular hypertensive rats. Am J Hypertens. 1991;4(10 Pt 1):815-819.

34. Gava AL, Peotta VA, Cabral AM, Vasquez EC, Meyrelles SS. Overexpression of eNOS prevents the development of renovascular hypertension in mice. Can J Physiol Pharmacol. 2008;86(7):458-464.

35. Wallace JM. Hemodynamic lesions in hypertension. Am J Cardiol. 1975;36(5):670-684.

36. Meredith IT, Friberg P, Jennings GL, et al. Exercise training lowers resting renal but not cardiac sympathetic activity in humans. Hypertension. 1991;18(5):575-582.

37. Navar LG, Mitchell KD, Harrison-Bernard LM, Kobori H, Nishiyama A. Intrarenal angiotensin II levels in normal and hypertensive states. J Renin Angiotensin Aldosterone Syst. 2001;2(1 Suppl):S176-S184.

38. Muller DN, Klanke B, Feldt S, et al. (Pro)renin receptor peptide inhibitor "handle-region" peptide does not affect hypertensive nephrosclerosis in Goldblatt rats. Hypertension. 2008;51(3):676-681.

39. Liu JL, Irvine S, Reid IA, Patel KP, Zucker IH. Chronic exercise reduces sympathetic nerve activity in rabbits with pacing-induced heart failure: a role for angiotensin II. Circulation. 2000;102(15):1854-1862.

40. Lincevicius GS, Shimoura CG, Nishi EE, et al. Aldosterone contributes to sympathoexcitation in renovascular hypertension. Am J Hypertens. 2015;28(9):1083-1090.

41. Mackenzie HS, Morrill AL, Ploth DW. Pressure dependence of exaggerated natriuresis in two-kidney, one clip Goldblatt hypertensive rats. Kidney Int. 1985;27(5):731-738.

42. Ploth DW, Kleeman K, Morrill L, Rademacher R, Jackson CA. Effects of verapamil and converting enzyme inhibition on bilateral renal function of two-kidney, one-clip hypertensive rats. Clin Sci (Lond). 1987;72(6):657-667.

43. Rademacher R, Berecek KH, Ploth DW. Effects of angiotensin inhibition and renal denervation in two-kidney, one clip hypertensive rats. Hypertension. 1986;8(12):1127-1134.

44. Guron GS, Grimberg ES, Basu S, Herlitz H. Acute effects of the superoxide dismutase mimetic tempol on split kidney function in two-kidney one-clip hypertensive rats. J Hypertens. 2006;24(2):387-394.

45. Cervenka L, Wang CT, Mitchell KD, Navar LG. Proximal tubular angiotensin II levels and renal functional responses to AT1 receptor blockade in nonclipped kidneys of Goldblatt hypertensive rats. Hypertension. 1999;33(1):102-107. 
46. Imamura A, Mackenzie HS, Lacy ER, Hutchison FN, Fitzgibbon WR, Ploth DW. Effects of chronic treatment with angiotensin converting enzyme inhibitor or an angiotensin receptor antagonist in two-kidney, one-clip hypertensive rats. Kidney Int. 1995;47(5):1394-1402.

47. Navar LG. Intrarenal renin-angiotensin system in regulation of glomerular function. Curr Opin Nephrol Hypertens. 2014;23(1):38-45.

48. Roman RJ, Cowley AW Jr. Characterization of a new model for the study of pressure-natriuresis in the rat. Am J Physiol. 1985;248(2 Pt 2):F190-F198.

49. Olsen ME, Hall JE, Montani JP, Guyton AC, Langford HG, Cornell JE. Mechanisms of angiotensin II natriuresis and antinatriuresis. Am J Physiol. 1985;249(2 Pt 2):F299-F307.

50. Lohmeier TE, Lohmeier JR, Reckelhoff JF, Hildebrandt DA. Sustained influence of the renal nerves to attenuate sodium retention in angiotensin hypertension. Am J Physiol Regul Integr Comp Physiol. 2001;281(2):R434-R443.

51. Winternitz SR, Katholi RE, Oparil S. Role of the renal sympathetic nerves in the development and maintenance of hypertension in the spontaneously hypertensive rat. J Clin Invest. 1980;66(5): 971-978.
52. Katholi RE, Whitlow PL, Winternitz SR, Oparil S. Importance of the renal nerves in established two-kidney, one clip Goldblatt hypertension. Hypertension. 1982;4(3 Pt 2):166-174.

53. Hropot M, Fowler N, Karlmark B, Giebisch G. Tubular action of diuretics: distal effects on electrolyte transport and acidification. Kidney Int. 1985;28(3):477-489.

54. Wei Y, Zavilowitz B, Satlin LM, Wang WH. Angiotensin II inhibits the ROMK-like small conductance $\mathrm{K}$ channel in renal cortical collecting duct during dietary potassium restriction. J Biol Chem. 2007;282(9):6455-6462.

55. Munoz-Pacheco P, Ortega-Hernandez A, Caro-Vadillo A, et al. Eplerenone enhances cardioprotective effects of standard heart failure therapy through matricellular proteins in hypertensive heart failure. J Hypertens. 2013;31(11):2309-2318; discussion 2319.

56. Ceravolo GS, Montezano AC, Jordao MT, et al. An interaction of renin-angiotensin and kallikrein-kinin systems contributes to vascular hypertrophy in angiotensin II-induced hypertension: in vivo and in vitro studies. PLoS One. 2014;9(11):e111117.

57. Clorius JH, Mandelbaum A, Hupp T, et al. Exercise activates renal dysfunction in hypertension. Am J Hypertens. 1996;9(7):653-661.
Integrated Blood Pressure Control

\section{Publish your work in this journal}

Integrated Blood Pressure Control is an international, peer-reviewed open-access journal focusing on the integrated approach to managing hypertension and risk reduction. Treating the patient and comorbidities together with diet and lifestyle modification and optimizing healthcare resources through a multidisciplinary team approach constitute key

\section{Dovepress}

features of the journal. This journal is indexed on American Chemical Society's Chemical Abstracts Service (CAS). The manuscript management system is completely online and includes a very quick and fair peerreview system, which is all easy to use. Visit http://www.dovepress.com/ testimonials.php to read real quotes from published authors.

Submit your manuscript here: https://www.dovepress.com/integrated-blood-pressure-control-journal 